\title{
CORRELATED RANDOM WALKS WITH STAY
}

\author{
Ram Lal* \\ U. Narayan Bhat \\ Department of Statistical Science \\ Southern Methodist University, Dallas, Texas 75275
}

\begin{abstract}
A random walk describes the movement of a particle in discrete time, with the direction and the distance traversed in one step being governed by a probability distribution. In a correlated random walk (CRW) the movement follows a Markov chain and induces correlation in the state of the walk at various epochs. Then, the walk can be modelled as a bivariate Markov chain with the location of the particle and the direction of movement as the two variables. In such random walks, normally, the particle is not allowed to stay at one location from one step to the next. In this paper we derive explicit results for the following characteristics of the CRW when it is allowed to stay at the same location, directly from its transition probability matrix: (i) equilibrium solution and the first passage probabilities for the CRW restricted on one side, and (ii) equilibrium solution and first passage characteristics for the CRW restricted on both sides (i.e., with finite state space).
\end{abstract}

Key words: Correlated random walk, Markov chain, equilibrium solution, first passage time.

AMS Classification: $60 \mathrm{~J} 15,60 \mathrm{~J} 40$

OR/MS Index Subject Classification: 569 Random Walk, 568 Markov

Processes

\section{INTRODUCTION}

A random walk describes the movement of a particle in discrete time, with the direction and the distance traversed in one step being governed by a probability

\footnotetext{
* Invited paper
} 
distribution. In a correlated random walk (CRW), the probability distribution at successive epochs of the walk is dependent on the preceding steps in a Markovian structure. Consequently, the walk itself becomes a correlated process. Starting with Gillis (1955) and Mohan (1955), several authors have investigated problems related to CRW's in one and more dimensions, and its special cases such as the symmetric CRW which has the same probability for proceeding in the same direction in successive epochs. All these investigations have used the classical approach of difference equations and generating functions. Except for Nain and Sen (1979a), the papers by Gupta (1958), Seth (1963), Jain (1971, 1973), Darroch and Whitford (1972), Proudfoot and Lampard (1972), Nain and Sen (1979b, 1980), Renshaw and Henderson (1981), Henderson et al. (1983, 1984), Bender and Richmond (1984) and Roerdink (1985) have considered a CRW in which the particle is not allowed to stay at the same location from one epoch to the next. Also, in most of these investigations first passage problems are of primary interest. In the lone investigation of an unrestricted CRW with stay, Nain and Sen (1979a) consider characteristics such as first passage to a specific state, return to the origin, passage to a state a given number of times, and transitions crossing the $x$-axis and rebounding from it. The results are mostly in terms of generating functions. In this investigation we propose to concentrate on the equilibrium distribution of the walk when restrictions are imposed on the state space on one or both sides and some characteristics such as the probability of first return into a set of contiguous states and first passage probabilities and mean passage times with respect to specified states. All results are either in explicit or in easily computable expressions.

The investigation is facilitated by representing the CRW by a bivariate process $\left\{\left(W_{n}, U_{n}\right), n=0,1,2, \ldots\right\}$, where $W_{n}$ is the location of the particle after $n$ steps and $U_{n}$ is the nature of the step signified by -1 for a move to the left, 0 for stay in the same state, and +1 for a move to the right. We consider the walk restricted to the non-negative integers, and thus we have a product space for the walk with factors

$$
\begin{aligned}
& W=(0,1,2, \ldots) \\
& U=(-1,0,+1) .
\end{aligned}
$$

The process $\left\{U_{n}\right\}$ determining the direction of the walk is a Markov chain with transition probability matrix 


$$
P_{U}=\quad \begin{array}{r}
+1 \\
0 \\
-1
\end{array}\left[\begin{array}{llc}
a & b & 1-a-b \\
c & d & 1-c-d \\
g & h & 1-g-h
\end{array}\right],
$$

$$
0 \leq \mathrm{a}, \mathrm{b}, \mathrm{c}, \mathrm{d}, \mathrm{g}, \mathrm{h} \leq 1 \text {. }
$$

We also assume that the elements are such that this Markov chain is irreducible and aperiodic. As $n \rightarrow \infty,\left\{U_{n}, n=0,1,2, \ldots\right\}$ has the following properties.

Let $\Pi=\left(\pi_{+1}, \pi_{0}, \pi_{-1}\right)$ be the limiting distribution of $U_{n}$. Using well-known properties of Markov chains $\left(\Pi P_{U}=\Pi\right.$ and $\left.\Pi e=1\right)$, we get

$$
\begin{aligned}
& \pi_{+1}=\frac{h c+(1-d) g}{(1-a)(1-d)-b c} \pi_{-1} \\
& \pi_{0}=\frac{b g+(1-a) h}{(1-d)(1-a)-b c} \pi_{-1}
\end{aligned}
$$

leading to the result

$$
\begin{aligned}
& \pi_{+1}=[\mathrm{hc}+(1-\mathrm{d}) \mathrm{g}] / \Phi \\
& \pi_{0}=[\mathrm{bg}+(1-\mathrm{a}) \mathrm{h}] / \Phi \\
& \pi_{-1}=[(1-\mathrm{a})(1-\mathrm{d})-\mathrm{bc}] / \Phi
\end{aligned}
$$

where

$$
\bar{\sigma}=(1-d)(1-a+g)+h(1-a+c)+b(g-c) .
$$

Assuming that $U_{0}$ has the distribution given by (1.3), the serial correlation with lag 1 of the process $\left\{U_{n}\right\}$ can be obtained as

$$
\rho=\left[(2 a+b-1) \pi_{+1}-(2 g+h-1) \pi_{-1}-[E(U)]^{2}\right] / V(U)
$$

where 


$$
\begin{array}{r}
\mathrm{E}(\mathrm{U})=\pi_{+1}-\pi_{-1}, \\
\mathrm{~V}(\mathrm{U})=\pi_{+1}+\pi_{-1}-[\mathrm{E}(\mathrm{U})]^{2} .
\end{array}
$$

The transition probability matrix of the CRW can now be given as follows.

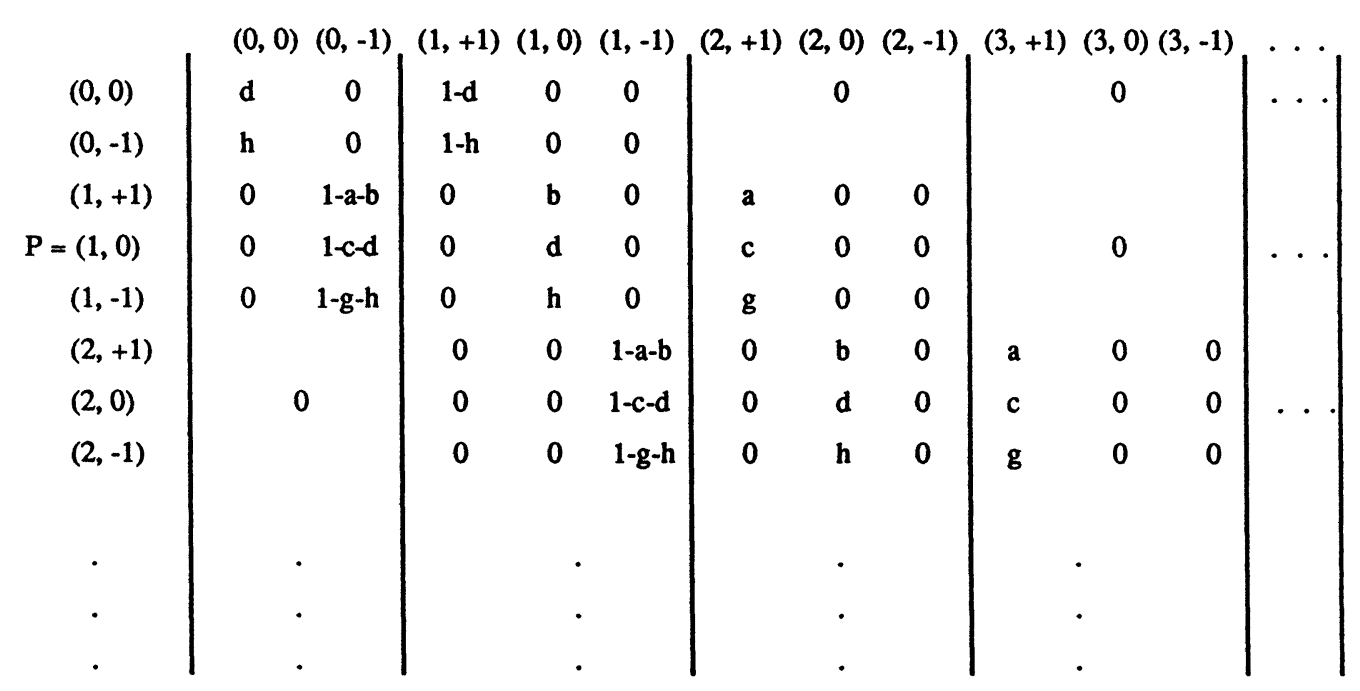

$$
\begin{aligned}
& 0 \\
& 0 \\
& 1 \\
& 2 \\
& 3 \\
& \vdots
\end{aligned}\left[\begin{array}{llllll}
\mathbf{A} & \mathrm{B} & & & & \\
\mathrm{C} & \mathrm{Z} & \mathrm{Y} & & \\
& \mathrm{X} & \mathrm{Z} & \mathrm{Y} & & \\
& & \mathrm{X} & \mathrm{Z} & \mathrm{Y} & \\
& & & & . &
\end{array}\right]
$$

where

$$
A=\left[\begin{array}{ll}
d & 0 \\
h & 0
\end{array}\right], B=\left[\begin{array}{ccc}
1-d & 0 & 0 \\
1-h & 0 & 0
\end{array}\right],
$$




$$
\begin{gathered}
C=\left[\begin{array}{ll}
0 & 1-a-b \\
0 & 1-c-d \\
0 & 1-g-h
\end{array}\right], X=\left[\begin{array}{lll}
0 & 0 & 1-a-b \\
0 & 0 & 1-c-d \\
0 & 0 & 1-g-h
\end{array}\right], \\
Y=\left[\begin{array}{lll}
a & 0 & 0 \\
c & 0 & 0 \\
g & 0 & 0
\end{array}\right], \quad Z=\left[\begin{array}{lll}
0 & b & 0 \\
0 & d & 0 \\
0 & h & 0
\end{array}\right] .
\end{gathered}
$$

Note that $\mathrm{P}_{\mathrm{U}}=\mathrm{X}+\mathrm{Y}+\mathrm{Z}$.

We shall exploit the structure of the submatrices defined in (1.6) and the transition probability matrix $\mathbf{P}$ to obtain explicit expressions for the distribution characteristic of the CRW.

In section 2 we obtain the limiting distribution of the CRW with transition probability matrix (1.5) and related first passage characteristics. In Section 3 similar properties are investigated for the CRW when the state space is made finite by placing restrictions on both sides.

\section{CRW RESTRICTED ON ONE SIDE}

The condition for the existence of an equilibrium solution is given by the following theorem.

Theorem 2.1. The necessary and sufficient condition for the Markov chain with the transition probability matrix (1.5) to have an equilibrium solution is given by

$$
\frac{a(1-d)+b c}{(1-d)(1-g)-h c}<1
$$

Proof. Following Neuts (1981, p. 32), the necessary and sufficient condition for the existence of the equilibrium solution may be stated as

$$
\text { mYe }<\text { חXe }
$$

where $\Pi$ is the limiting distribution of the Markov chain $\left\{U_{n}\right\}$ as given in (1.3). Going back to the equations $\Pi P_{U}=\Pi$, i.e., $\Pi(X+Y+Z)=\Pi$, we observe, 
(2.3)

$$
\Pi \mathrm{Ye}=\pi_{+1}
$$

$$
\text { חXe }=\pi_{-1}
$$

Thus, the condition (2.2) reduces to

$$
\pi_{+1}<\pi_{-1}
$$

Substituting from (1.3), we get

$$
h c+(1-d) g<(1-a)(1-d)-b c,
$$

which on simplification reduces to the condition (2.1).

Let

$$
x=\left(x_{0}, x_{1}, x_{2}, \ldots\right)
$$

where $x_{0}$ is a two element row vector and $x_{i}(i=1,2, \ldots)$ are three element row vectors, be the limiting distribution of the Markov chain. Following Neuts (1981, p. 25) we have

$$
x_{i}=x_{1} R^{i-1}, i=1,2, \ldots,
$$

where $R$ is the rate matrix of the Markov chain $P$. It is obtained in the following lemma.

Lemma 2.1

where

$$
\begin{aligned}
R & =\left[\begin{array}{lll}
a & a \alpha & a \beta \\
c & c \alpha & c \beta \\
g & g \alpha & g \beta
\end{array}\right], \\
R^{j} & =\beta^{j-1} R, \quad j=1,2,3 \ldots,
\end{aligned}
$$

$$
\alpha=\frac{a h+b(1-g)}{(1-d)(1-g)-h c}, \beta=\frac{b c+a(1-d)}{(1-d)(1-g)-h c} .
$$

Proof. The rate matrix $\mathrm{R}$ satisfies the matrix equation

$$
R=Y+R Z+R^{2} X
$$

which can be written as

$$
R=Y(I-Z)^{-1}+R^{2} X(I-Z)^{-1}
$$


Let $R_{n}$ be the $n^{\text {th }}$ iterate of $R$, such that

$$
R_{n}=Y(I-Z)^{-1}+R_{n-1}^{2} X(I-Z)^{-1}
$$

We have

$$
(I-Z)^{-1}=\left[\begin{array}{lll}
1 & b /(1-d) & 0 \\
0 & 1 /(1-d) & 0 \\
0 & h /(1-d) & 1
\end{array}\right]
$$

Substituting into (2.11) we get

$$
R_{n}=R_{n-1}^{2}\left[\begin{array}{ccc}
0 & \frac{h(1-a-b)}{1-d} & 1-a-b \\
0 & \frac{h(1-c-d)}{1-d} & 1-c-d \\
0 & \frac{h(1-g-h)}{1-d} & 1-g-h
\end{array}\right]+\left[\begin{array}{ccc}
a & \frac{a b}{1-d} & 0 \\
c & \frac{c b}{1-d} & 0 \\
g & \frac{g b}{1-d} & 0
\end{array}\right] \text {. }
$$

By induction we can show that, for $\mathrm{n} \geq 1$,

$$
R_{n}=\left[\begin{array}{ccc}
a & \frac{a}{1-d}\left(b+h K_{n}\right) & a K_{n} \\
c & \frac{c}{1-d}\left(b+h K_{n}\right) & c K_{n} \\
g & \frac{g}{1-d}\left(b+h K_{n}\right) & g K_{n}
\end{array}\right]
$$

where $K_{n}$ satisfies the recurrence relation

$$
\begin{aligned}
& K_{n}=\left[a+\frac{b c}{1-d}+\left(g+\frac{h c}{1-d}\right) K_{n-1}\right]\left[1-a-\frac{b c}{1-d}+\left(1-g-\frac{h c}{1-d}\right) K_{n-1}\right] \\
& K_{1}=0 .
\end{aligned}
$$

As $n \rightarrow \infty, R^{n} \rightarrow R$; hence $K_{n} \rightarrow K$ as well. Thus $K$ can be determined by solving 


$$
K=\left[a+\frac{b c}{1-d}+\left(g+\frac{h c}{1-d}\right) K\right]\left[1-a \frac{b c}{1-d}+\left(1-g-\frac{h c}{1-d}\right) K\right]
$$

or

$$
\begin{aligned}
0= & \left(a+\frac{b c}{1-d}\right)\left(1-a-\frac{b c}{1-d}\right)+\left[\left(a+\frac{b c}{1-d}\right)\left(1-g-\frac{h c}{1-d}\right)-1\right. \\
& \left.+\left(1-a-\frac{b c}{1-d}\right)\left(g+\frac{h c}{1-d}\right)\right] K+\left(g+\frac{h c}{1-d}\right)\left(1-g-\frac{h c}{1-d}\right) K^{2} .
\end{aligned}
$$

After simplification the discriminant of equation (2.15) can be written as

$$
=\left[\frac{c(b+h)}{1-d}+a+g-1\right]^{2}
$$

and the two possible roots of the equation are

$$
K=\frac{a(1-d)+b c}{(1-g)(1-d)-h c}, \frac{(1-a)(1-d)-b c}{g(1-d)+h c}
$$

From (2.6) we may note that $\mathrm{R}^{\mathrm{j}} \rightarrow 0$ as $\mathrm{j} \rightarrow \infty$. Using this condition we can show that the second solution is inadmissible. The lemma now follows using the first solution in (2.17) and simplifying.

We may also note that the condition (2.1) is necessary for $R^{j} \rightarrow 0$ as $j \rightarrow \infty$.

Using $\mathbf{R}$, the equilibrium solution is determined in the following theorem.

Theorem 2.2 When

$$
\frac{a(1-d)+b c}{(1-d)(1-g)-h c}<1,
$$

the limiting distribution of $a C R W$ is given by

$$
x=\left(x_{0}, x_{1}, x_{2}, \ldots\right),
$$

where 


$$
\begin{gathered}
x_{0}=\frac{(1-d)(1-a-g)-c(h+b)}{((1-d)(1-g)-h c)(2(1-d)+h+b)}[h,(1-d)], \\
x_{j}=\frac{(1-d)((1-d)(1-a-g)-c(h+b))}{((1-d)(1-g)-h c)(2(1-d)+h+b)}\left[\frac{a(1-d)+b c}{(1-d)(1-g)-h c}\right]^{j-1} \\
{\left[1, \frac{b(1-g)+a h}{(1-d)(1-g)-h c}, \frac{a(1-d)+b c}{(1-d)(1-g)-h c}\right],} \\
j=1,2, \ldots
\end{gathered}
$$

Proof. Writing out $\mathrm{xP}=\mathrm{x}$, we have

$$
\begin{aligned}
& x_{0} A+x_{1} C=x_{0} \\
& x_{0} B+x_{1} Z+x_{2} X=x_{1} \\
& x_{i-1} Y+x_{i} Z+x_{i+1} X=x_{i} \quad i \geq 2
\end{aligned}
$$

From the second equation in (2.20) and equation (2.6) we get

$$
x_{1}=x_{0} B(I-Z-R X)^{-1}
$$

and

$$
x_{j}=x_{0} B(I-Z-R X)^{-1} R^{j-1}, j \geq 1
$$

From the first equation in (2.20) and the normalizing condition $x e=1$, we see that $x_{0}$ is the solution of the following reduced system

$$
\begin{aligned}
& x_{0}\left(A+B(I-Z-R X)^{-1} C\right)=x_{0} \\
& x_{0} e+x_{0} B(I-Z-R X)^{-1}(I-R)^{-1} e=1
\end{aligned}
$$

where we have assumed that the unit column vectors e have appropriate dimensions.

Substituting from (1.6) and (2.7) for A, B, C, X, Z and R and simplifying we get the following results 


$$
(I-Z-R X)^{-1}=\left[\begin{array}{ccc}
1 & (b(1-g)+a h) \eta & (a(1-d)+b c) \eta \\
0 & (1-g) \eta & c \eta \\
0 & h \eta & (1-d) \eta
\end{array}\right]
$$

where

$$
\eta=((1-\mathrm{d})(1-\mathrm{g})-\mathrm{hc})^{-1}
$$

$$
\begin{gathered}
A+B(I-Z-R X)^{-1} C=\left[\begin{array}{ll}
d & 1-d \\
h & 1-h
\end{array}\right] \\
(I-R)^{-1}=I+\frac{(1-d)(1-g)-h c}{(1-d)(1-a-g)-c(h+b)} R \\
B(I-Z-R X)^{-1}(I-R)^{-1}=\frac{(1-d)(1-g)-h c}{(1-d)(1-a-g)-c(h+b)} B(I-Z-R X)^{-1} \\
B(I-Z-R X)^{-1}(I-R)^{-1} e=\frac{(1-d+b)(1-g+a)+(h-b)(a-c)}{(1-d)(1-a-g)-c(h+b)}\left[\begin{array}{l}
1-d \\
1-h
\end{array}\right] .
\end{gathered}
$$

The results (2.18) and (2.19) now follow after we substitute these results into (2.22) and (2.23) and simplify.

We close this section by giving a simple result on a first passage problem. Consider two complementary sets of states

$$
\begin{aligned}
& S_{i}=\{(0,0),(0,-1),(1,+1),(1,0),(1,-1), \ldots,(i,+1),(i, 0),(i,-1)\} \\
& S_{i}^{c}=\{(i+1,+1),(i+1,0),(i+1,-1), \ldots\} .
\end{aligned}
$$

Let ${ }_{i} P_{i i}$ be the first return probability to set $S_{i}$ (i.e., the first passage probability of the process to states in the set $S_{i}$, after visiting states in $S_{i}^{c}$ while avoiding states in $S_{i}$, having left $S_{i}$ initially). We have 
Theorem 2.3

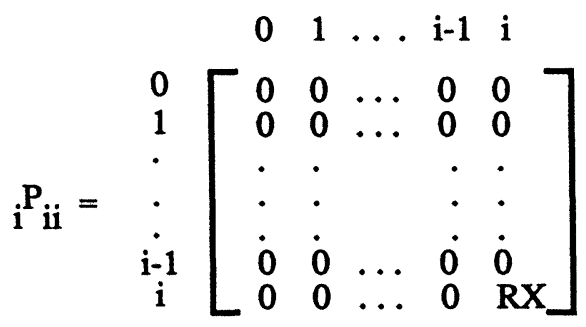

where

$$
\mathrm{RX}=\left[\begin{array}{lll}
0 & 0 & \mathrm{a} \\
0 & 0 & \mathrm{c} \\
0 & 0 & \mathrm{~g}
\end{array}\right]
$$

Proof. When a transition probability matrix is partitioned as

$$
\mathrm{P}=\mathrm{y}_{1}\left[\begin{array}{cc}
\mathrm{y}_{1} & \mathrm{y}_{2} \\
\mathrm{~A}_{2} & \mathrm{~B}^{*} \\
\mathrm{C}^{*} & \mathrm{D}^{*}
\end{array}\right],
$$

it is well known that the elements of the matrix $B^{*}\left(I-D^{*}\right)^{-1} C^{*}$ give the first return probability (in the sense described above) into states $y_{1}$. If $x_{1}^{*}$ and $x_{2}^{*}$ are the limiting probabilities corresponding to sets of states $y_{1}$ and $y_{2}$, using reduced systems (see Lal and Bhat $(1987,1988))$ we get the relation

$$
x_{2}^{*}=x_{1}^{*} B^{*}\left(1-D^{*}\right)^{-1}
$$

Using the rate matrix $R$ and equation (2.6), we can write (2.31) as

$$
x_{2}^{*}=x_{1}^{*}\left[\begin{array}{cccc}
0 & 0 & \ldots \\
0 & 0 & \ldots & \ldots \\
\cdots & & \\
\cdots & & \\
R & R^{2} & &
\end{array}\right]
$$


Comparing (2.31) with (2.32) and noting that

$$
\mathrm{C}^{*}=\left[\begin{array}{cccc}
0 & 0 & \ldots & \mathrm{X} \\
0 & 0 & \ldots & 0 \\
\cdot & \cdot & & \cdot \\
\cdot & \cdot & & \cdot \\
\dot{0} & 0 & \ldots & 0
\end{array}\right]
$$

we get the result in the theorem.

When $S_{i}=\{(0,0),(0,-1)\}$, for $B^{*}\left(I-D^{*}\right)^{-1} C^{*}$ we have $B(I-Z-R X)^{-1} C$, which simplifies to

$$
\left[\begin{array}{ll}
0 & 1-d \\
0 & 1-h
\end{array}\right]
$$

When we combine these probabilities with the elements of

$$
A=\left[\begin{array}{ll}
d & 0 \\
h & 0
\end{array}\right]
$$

we find, as we should, that the recurrence probabilities to the these states are 1. Further, using arguments similar to those used above, we can show that $B(I-Z-R X)^{-1} R^{j-1}$ gives the expected number of visits to states $\{(j,+1),(j, 0),(j,-1)\}$ before first passage to $\{(0,0),(0,-1)\}$ while avoiding it in the meantime, after having started initially from it. We have

$$
B(I-Z-R X)^{-1} R^{j-1}=\beta^{j-1}\left[\begin{array}{lll}
1-d & (1-d) \alpha & (1-d) \beta \\
1-h & (1-h) \alpha & (1-h) \beta
\end{array}\right],
$$

where $\alpha$ and $\beta$ are given in (2.9). Thus, the mean recurrence time of the set $\{(0,0),(0,-1)\}$ is obtained as 


$$
\begin{aligned}
& \frac{1}{1-\beta}\left[\begin{array}{l}
(1-d)(1+\alpha+\beta) \\
(1-h)(1+\alpha+\beta)
\end{array}\right] \\
= & \frac{(1-d+b)(1-g+a)+(h-b)(a-c)}{(1-d)(1-a-g)-c(h+b)}\left[\begin{array}{c}
1-d \\
1-h
\end{array}\right] .
\end{aligned}
$$

\section{CRW RESTRICTED ON BOTH SIDES}

Let the walk be restricted to the states $\{0,1,2, \ldots, N\}$. Then, the transition probability matrix takes the form

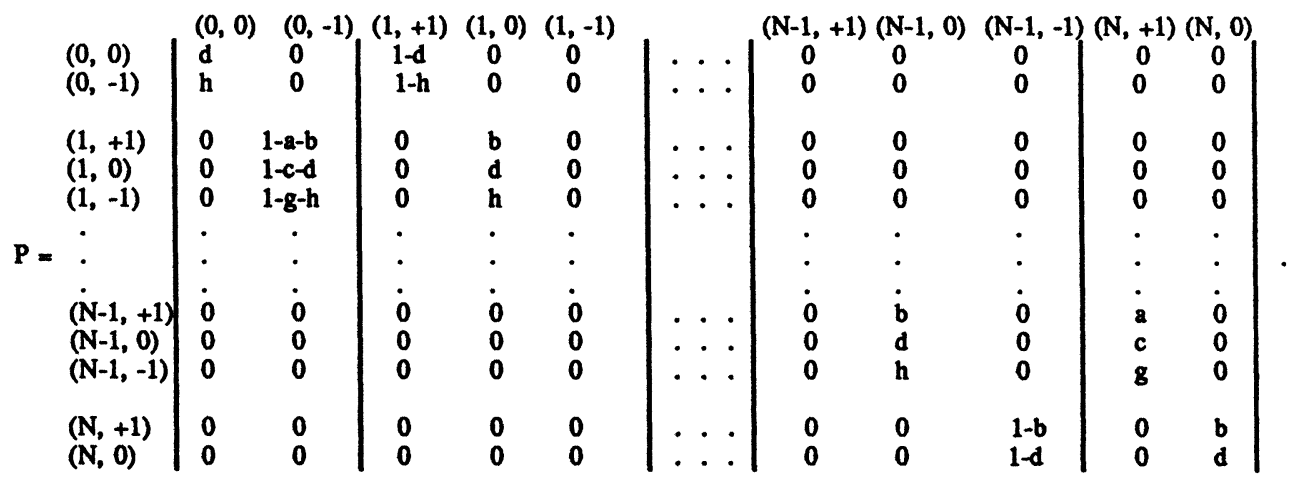

Using the submatrices defined in (1.6) and

$$
\begin{gathered}
E=\left[\begin{array}{ll}
0 & b \\
0 & d
\end{array}\right], \\
F=\left[\begin{array}{lll}
0 & 0 & 1-b \\
0 & 0 & 1-d
\end{array}\right], \text { and } G=\left[\begin{array}{ll}
a & 0 \\
c & 0 \\
g & 0
\end{array}\right],
\end{gathered}
$$

we may represent $\mathbf{P}$ as 
(3.1)

$$
\mathrm{P}=\begin{array}{l|llllllll}
0 & 0 & 1 & 2 & 3 & \ldots & \mathrm{N}-2 & \mathrm{~N}-1 & \mathrm{~N} \\
1 & \mathrm{~A} & \mathrm{~B} & 0 & 0 & \ldots & 0 & 0 & 0 \\
2 & \mathrm{C} & \mathrm{Z} & \mathrm{Y} & 0 & \ldots & 0 & 0 & 0 \\
. & \mathrm{X} & \mathrm{Z} & \mathrm{Y} & \ldots & 0 & 0 & 0 \\
. & . & . & . & & . & . & . \\
. & . & . & . & & . & . & . \\
\mathrm{N}-2 & 0 & 0 & 0 & 0 & \ldots & \mathrm{Z} & \mathrm{Y} & 0 \\
\mathrm{~N}-1 & 0 & 0 & 0 & 0 & \ldots & \mathrm{X} & \mathrm{Z} & \mathrm{G} \\
\mathrm{N} & 0 & 0 & 0 & 0 & \ldots & 0 & \mathrm{~F} & \mathrm{E}
\end{array} \mid .
$$

The limiting distribution $x=\left(x_{0}, x_{1}, x_{2}, \ldots, x_{N}\right)$ of the CRW can be determined by our using a backward recursion (see Lal and Bhat, 1987, 1988) as in the following theorem. Note that

$$
\begin{aligned}
& x_{0}=\left(x_{00}, x_{0,-1}\right), \\
& x_{i}=\left(x_{i,+1}, x_{i, 0}, x_{i,-1}\right), i=1,2, \ldots, N-1, \\
& x_{N}=\left(x_{N,+1}, x_{N, 0}\right) .
\end{aligned}
$$

Theorem 3.1

$$
\begin{aligned}
& x_{00}=\left[1+\frac{b}{h} \beta^{N-1}+\frac{1-d}{h}\left(2 \frac{1-\beta^{N}}{1-\beta}+\beta \frac{1-\beta^{N-1}}{1-\beta}\right)\right]^{-1}, \\
& x_{i,+1}=\left(\frac{1-d}{h}\right) \beta^{i-1} x_{00}, \quad i=1,2, \ldots, N,
\end{aligned}
$$


(3.2) Continued

$$
\begin{array}{ll}
x_{i, 0}=\left(\frac{1-d}{h}\right) \alpha \beta^{i-1} x_{00}, & i=1,2, \ldots, N-1, \\
x_{i,-1}=\left(\frac{1-d}{h}\right) \beta^{i} x_{00}, & i=0,1,2, \ldots, N-1, \\
x_{N, 0}=\left(\frac{b}{h}\right) \beta^{N-1} x_{00}, &
\end{array}
$$

where

$$
\alpha=\frac{a h+b(1-g)}{(1-d)(1-g)-h c} \text { and } \beta=\frac{b c+a(1-d)}{(1-d)(1-g)-h c}
$$

as defined in (2.9).

Proof. Clearly we have

$$
\begin{aligned}
& x P=x \\
& x e=1 .
\end{aligned}
$$

To use the backward recursion, we start with the last 4 equations in (3.5).

$$
\begin{aligned}
& x_{N, 0}=b x_{N,+1}+d x_{N, 0} \\
& x_{N,+1}=a x_{N-1,+1}+c x_{N-1,0}+g x_{N-1,-1} \\
& x_{N-1,-1}=(1-b) x_{N,+1}+(1-d) x_{N, 0} \\
& x_{N-1,0}=b x_{N-1,+1}+d x_{N-1,0}+h x_{N-1,-1} .
\end{aligned}
$$

From these equations, the last 4 variables can be expressed in terms of $x_{N-1,+1}$ as

$$
\begin{aligned}
& x_{N, 0}=\frac{b}{1-d} \beta x_{N-1,+1} \\
& x_{N,+1}=\beta x_{N-1,+1}
\end{aligned}
$$

$$
\begin{aligned}
& x_{\mathrm{N}-1,-1}=\beta \mathrm{x}_{\mathrm{N}-1,+1} \\
& \mathrm{x}_{\mathrm{N}-1,0}=\alpha \mathrm{x}_{\mathrm{N}-1,+1}
\end{aligned}
$$


where $\alpha$ and $\beta$ are defined in (3.4). Next we use three equations preceding the last 4 in (3.5):

$$
\begin{aligned}
& \mathrm{x}_{\mathrm{N}-1,+1}=\mathrm{ax}_{\mathrm{N}-2,+1}+\mathrm{cx} \mathrm{N}-2,0_{\mathrm{N}-2,-1}+\mathrm{gx} \\
& \mathrm{x}_{\mathrm{N}-2,-1}=(1-\mathrm{a}-\mathrm{b}) \mathrm{x}_{\mathrm{N}-1,+1}+(1-\mathrm{c}-\mathrm{d}) \mathrm{x}_{\mathrm{N}-1,0}+(1-\mathrm{g}-\mathrm{h}) \mathrm{x}_{\mathrm{N}-1,-1} \\
& \mathrm{x}_{\mathrm{N}-2,0}=\mathrm{bx} \mathrm{N}-2,+1_{\mathrm{N}-2,0}+\mathrm{hx} \mathrm{x}_{\mathrm{N}-2,-1} .
\end{aligned}
$$

Now expressing all the variables in terms $x_{N-2,+1}$ and using (3.7), from these equations we get

$$
\begin{aligned}
& x_{N 0}=\left(\frac{b}{1-d}\right) \beta^{2} x_{N-2,+1} \\
& x_{N,+1}=\beta^{2} x_{N-2,+1} \\
& x_{N-1,-1}=\beta^{2} x_{N-2,+1} \\
& x_{N-1,0}=\alpha \beta x_{N-2,+1} \\
& x_{N-1,+1}=\beta x_{N-2,+1} \\
& x_{N-2,-1}=\beta x_{N-2,+1} \\
& x_{N-2,0}=\alpha x_{N-2,+1} .
\end{aligned}
$$

By repeatedly using the above recursive procedure we get

$$
\begin{array}{ll}
x_{N, 0}=\left(\frac{b}{1-d}\right) \beta^{N-1} x_{1,+1} & \\
x_{i, 0}=\alpha \beta^{i-1} x_{1,+1} & i=1,2, \ldots, N-1
\end{array}
$$

$$
\begin{array}{ll}
x_{i,+1}=\beta^{i-1} x_{1,+1} & i=1,2, \ldots, N \\
x_{i,-1}=\beta^{i} x_{1,+1} & i=0,1,2, \ldots, N-1
\end{array}
$$




$$
x_{1,+1}=\left(\frac{1-d}{h}\right) x_{00}
$$

The theorem now follows when we use (3.9) in the normalizing condition (3.6).

In random walk problems first passage problems are also of interest. For instance, in the context of a gambler's ruin problem, the probability of ruin, the probability of win and the expected duration of the game are all related to the first passage of the walk to a particular state. In the case of a CRW with stay similar problems can also be investigated. A computational procedure to obtain these characteristics is to determine what has come to be known as the fundamental matrix of the appropriate transition probability matrix. See Kemeny and Snell (1960) for the definition of the matrix and the interpretation of results. The basic fundamental matrix for the finite state CRW can be given as

$$
\mathrm{M}=\left|\begin{array}{ccccccc}
1 & 2 & 3 & \cdots & \mathrm{N}-2 & \mathrm{~N}-1 & \mathrm{~N} \\
\mathrm{I}-\mathrm{Z} & -\mathrm{Y} & 0 & \ldots & 0 & 0 & 0 \\
-\mathrm{X} & \mathrm{I}-\mathrm{Z} & -\mathrm{Y} & \ldots & 0 & 0 & 0 \\
0 & -\mathrm{X} & \mathrm{I}-\mathrm{Z} & \ldots & 0 & 0 & 0 \\
. & . & . & & . & . & . \\
. & . & . & & . & . & . \\
. & . & . & & . & . & . \\
0 & 0 & 0 & \ldots & -\mathrm{X} & \mathrm{I}-\mathrm{Z} & -\mathrm{Y} \\
0 & 0 & 0 & \cdots & 0 & -\mathrm{X} & \mathrm{I}-\mathrm{Z}
\end{array}\right|^{-1}
$$

Let $M_{i j}$ be the $(i, j)$ th element of $M$. We have

Lemma 3.1

$$
M_{i j}=\sum_{t=0}^{\min (i-1, j-1)}\left(\prod_{q=t+1}^{i-1} X_{N-q}\right) R_{N-t}\left(\prod_{s=t+1}^{j-1} Y_{N-s}\right)
$$


where $R_{t}$ satisfies the relation

$$
\begin{aligned}
& R_{t}=\left(I-Z-Y R_{t-1} X\right)^{-1}, t=2,3, \ldots, N \\
& R_{1}=(I-Z)^{-1}, R_{0}=0
\end{aligned}
$$

and

$$
Y_{t}=Y_{t} \text { and } X_{t}=R_{t} X, t=1,2, \ldots, N
$$

In (3.11) $\prod_{\mathrm{q}=\mathrm{t}+1}^{\mathrm{i}-1} \mathrm{X}_{\mathrm{N}-\mathrm{q}}$ is taken in pre-order as q increases, while $\prod_{\mathrm{s}=\mathrm{t}+1}^{\mathrm{j}-1} \mathrm{Y}_{\mathrm{N}-\mathrm{s}}$

is taken in post-order as $s$ increases.

Proof. The result follows from the recursive algorithm following Theorem 2.2 of $\mathrm{Lal}$ and Bhat (1988).

Theorem 3.2

$$
\mathbf{M}_{\mathrm{ij}}=\left[\begin{array}{lll}
\mu_{\mathrm{ij}}^{(1)} & \mu_{\mathrm{ij}}^{(12)} & \mu_{\mathrm{ij}}^{(13)} \\
\mu_{\mathrm{ij}}^{(21)} & \mu_{\mathrm{ij}}^{(22)} & \mu_{\mathrm{ij}}^{(23)} \\
\mu_{\mathrm{ij}}^{(31)} & \mu_{\mathrm{ij}}^{(32)} & \mu_{\mathrm{ij}}^{(33)}
\end{array}\right],
$$

where

$$
\begin{aligned}
& \mu_{i j}^{(11)}=S_{i j}^{0}+\gamma S_{i j}^{5}+v s_{i j}^{7} \\
& \mu_{i j}^{(12)}=S_{i j}^{4}+h K_{N-i+1} s_{i j}^{6}+\left[\frac{b+(a h-b g) K_{N-j}}{i-d-v K_{N-j}}\right]\left(g s_{i j}^{5}+v s_{i j}^{7}\right) \\
& \mu_{i j}^{(13)}=\gamma s_{i j}^{2}+(1-d) K_{N-i+1} s_{i j}^{6}+\left[\frac{\gamma K_{N-j}}{1-d-v K_{N-j}}\right]\left(g s_{i j}^{5}+v s_{i j}^{7}\right) \\
& \mu_{j}^{(21)}=c s_{i j}^{5}+\gamma s_{i j}^{7}\left[1+\frac{c\left(K_{N-i}^{-1)}\right.}{1-d-v K_{N-i}}\right]
\end{aligned}
$$




$$
\begin{aligned}
& \mu_{\mathrm{ij}}^{(22)}=\mathrm{S}_{\mathrm{ij}}^{3}+h S_{\mathrm{ij}}^{6}\left[1+\frac{\mathrm{c}\left(\mathrm{K}_{\mathrm{N}-\mathrm{i}}-1\right)}{1-\mathrm{d}-\mathrm{v} \mathrm{K}_{\mathrm{N}-\mathrm{i}}}\right] \\
& +\left[\frac{\mathrm{b}+(\mathrm{ah}-\mathrm{bg}) \mathrm{K}_{\mathrm{N}-\mathrm{j}}}{1-\mathrm{d}-\mathrm{v} \mathrm{K}_{\mathrm{N}-\mathrm{j}}}\right]\left[\mathrm{cS}_{\mathrm{ij}}^{5}+v S_{\mathrm{ij}}^{7}\left(1+\frac{\mathrm{c}\left(\mathrm{K}_{\mathrm{N}-\mathrm{i}}-1\right)}{1-\mathrm{d}-\mathrm{v} \mathrm{K}_{\mathrm{N}-\mathrm{i}}}\right)\right] \\
& \mu_{\mathrm{ij}}^{(23)}=c S_{\mathrm{ij}}^{2}+(1-\mathrm{d}) \mathrm{S}_{\mathrm{ij}}^{6}\left[\frac{\mathrm{c}\left(\mathrm{K}_{\mathrm{N}-\mathrm{i}}-1\right)}{1-\mathrm{d}-v \mathrm{~K}_{\mathrm{N}-\mathrm{i}}}\right] \\
& +\left[\frac{\gamma \mathrm{K}_{\mathrm{N}-\mathrm{j}}}{1-\mathrm{d}-\mathrm{vK} \mathrm{K}_{\mathrm{N}-\mathrm{j}}}\right]\left[\mathrm{cS}_{\mathrm{ij}}^{5}+v S_{\mathrm{ij}}^{7}\left(1+\frac{\mathrm{c}\left(\mathrm{K}_{\mathrm{N}-\mathrm{i}}-1\right)}{1-\mathrm{d}-\mathrm{vK} \mathrm{K}_{\mathrm{N}-\mathrm{i}}}\right)\right] \\
& \mu_{i j}^{(31)}=v\left[S_{i j}^{5}+S_{i j}^{7}\left(\frac{1-d-v}{1-d-v K_{N-i}}\right)\right] \\
& \mu_{\mathrm{ij}}^{(32)}=h\left[S_{\mathrm{ij}}^{1}+S_{\mathrm{ij}}^{6}\left(\frac{1-\mathrm{d}-\mathrm{v}}{1-\mathrm{d}-\mathrm{vK} \mathrm{K}_{\mathrm{N}-\mathrm{i}}}\right)\right] \\
& +v\left[\frac{\mathrm{b}+(\mathrm{ah}-\mathrm{bg}) \mathrm{K}_{\mathrm{N}-\mathrm{j}}}{1-\mathrm{d}-\mathrm{vK} \mathrm{K}_{\mathrm{N}-\mathrm{j}}}\right]\left[\mathrm{s}_{\mathrm{ij}}^{5}+\mathrm{s}_{\mathrm{ij}}^{7}\left(\frac{1-\mathrm{d}-\mathrm{v}}{1-\mathrm{d}-\mathrm{vK} \mathrm{K}_{\mathrm{N}-\mathrm{i}}}\right)\right] \\
& \mu_{i j}^{(33)}=(1-d)\left[s_{i j}^{1}+s_{i j}^{6}\left(\frac{1-d-v}{1-d-v K_{N-i}}\right)\right] \\
& +v\left[\frac{v K_{N-j}}{1-d-v K_{N-j}}\right]\left[s_{i j}^{5}+S_{i j}^{7}\left(\frac{1-d-v}{1-d-v K_{N-i}}\right)\right]
\end{aligned}
$$

with

$$
\gamma=b c+a(1-d)
$$

where $\mathrm{K}_{\mathrm{t}}$ is defined by the recursion

$$
v=h c+g(1-d),
$$

$$
\mathrm{K}_{\mathrm{t}}=1+\frac{\gamma\left(\mathrm{K}_{\mathrm{t}-1}-1\right)}{1-\mathrm{d}-\mathrm{v} \mathrm{K}_{\mathrm{t}-1}}, \quad \mathrm{t}=1,2, \ldots .
$$

$$
\mathrm{K}_{0}=0 \text {, }
$$

and 


$$
\begin{aligned}
& S_{i j}^{0}=\sum_{0}^{I J} \delta_{i, t+2}^{* *} \delta_{j, t+2}^{* *} \\
& S_{i j}^{1}=\sum_{0}^{I J} \delta_{i, t+2}^{* *} \delta_{j, t+2}^{* *}\left(1-d-v K_{N-t-1}\right)^{-1}
\end{aligned}
$$$$
S_{i j}^{2}=\sum_{0}^{I J} \delta_{i, t+2}^{* *} \delta_{j, t+2}^{* *}\left(1-d-v K_{N-t-1}\right)^{-1} K_{N-t-1}
$$$$
S_{i j}^{3}=\sum_{0}^{I J} \delta_{i, t+2}^{* *} \delta_{j, t+2}^{* *}\left(1-d-v K_{N-t-1}\right)^{-1}\left(1-g K_{N-t-1}\right)
$$$$
S_{i j}^{4}=\sum_{0}^{I J} \delta_{i, t+2}^{* *} \delta_{j, t+2}^{* *}\left(1-d-v K_{N-t-1}\right)^{-1}\left(b+(a h-b g) K_{N-t-1}\right)
$$$$
S_{i j}^{5}=\sum_{0}^{I J} \delta_{i, t+2}^{* *} \delta_{t, j-1}^{* *}\left(1-d-v K_{N-t-1}\right)^{-1} k_{j-t-2}^{2}
$$$$
S_{i j}^{6}=\sum_{0}^{\mathrm{J}} \delta_{\mathrm{t}, \mathrm{i}-1}^{* *} \delta_{\mathrm{j}, \mathrm{t}+2}^{* *}\left(1-\mathrm{d}-\mathrm{vK_{ \textrm {N } - \mathrm { t } - 1 }}\right)^{-1} \mathrm{k}_{\mathrm{i}-\mathrm{t}-2}^{1}
$$

$$
S_{i j}^{7}=\sum_{0}^{I J} \delta_{t, i-1}^{* *} \delta_{t, j-1}^{* *}\left(1-d-v K_{N-t-1}\right)^{-1} \kappa_{i-t-2}^{1} \kappa_{j-t-2}^{2},
$$

where $\mathrm{IJ}=\min (\mathrm{i}-1, \mathrm{j}-1), \delta_{\mathrm{jk}}^{* *}=1$ if $\mathrm{j}<\mathrm{k}$, and $=0$ if $\mathrm{j} \geq \mathrm{k}$, and

$$
\begin{aligned}
& k_{T}^{1}=\gamma^{r}\left[\prod_{q=1}^{r}\left(1-d-v K_{N-i-q}\right)\right]^{-1} \\
& k_{r}^{2}=(1-d-v)^{r}\left[\prod_{q=1}^{r}\left(1-d-v K_{N-i-q}\right)\right]^{-1}
\end{aligned}
$$

Proof. From (3.12), setting 


$$
R_{t}=\left[\begin{array}{ccc}
r_{1}^{(t)} & r_{2}^{(t)} & r_{3}^{(t)} \\
r_{4}^{(t)} & r_{5}^{(t)} & r_{6}^{(t)} \\
r_{7}^{(t)} & r_{8}^{(t)} & r_{9}^{(t)}
\end{array}\right],
$$

we get after simplification

$$
R_{t}=\left[\begin{array}{ccc}
1 & -b & -a K_{t-1} \\
0 & 1-d & -c K_{t-1} \\
0 & -h & 1-g K_{t-1}
\end{array}\right]^{-1},
$$

where we have written

$$
K_{t}=(1-a-b) r_{1}^{(t)}+(1-c-d) r_{2}^{(t)}+(1-g-h) r_{3}^{(t)}
$$

Upon inversion (3.19) gives

$$
R_{t}=\left[\begin{array}{ccc}
1 & \frac{b+(a h-b g) K_{t-1}}{1-d-v K_{t-1}} & \frac{\gamma K_{t-1}}{1-d-v K_{t-1}} \\
0 & \frac{1-g K_{t-1}}{1-d-v K_{t-1}} & \frac{c K_{t-1}}{1-d-v K_{t-1}} \\
0 & \frac{h}{1-d-v K_{t-1}} & \frac{1-d}{1-d-v K_{t-1}}
\end{array}\right] .
$$

We also find that, for $t \geq 1, K_{t}$ satisfies the recursion given by (3.16).

Substituting from (3.20) in (3.13), we get 


$$
Y_{t}=\left[\begin{array}{ccc}
a & a \phi_{t-1}^{(1)} & a \phi_{t-1}^{(2)} \\
c & c \phi_{t-1}^{(1)} & c \phi_{t-1}^{(2)} \\
g & g \phi_{t-1}^{(1)} & g \phi_{t-1}^{(2)}
\end{array}\right]
$$

$$
X_{t}=\left[\begin{array}{ccc}
0 & 0 & K_{t} \\
0 & 0 & 1+\frac{c\left(K_{t-1}-1\right)}{1-d-v K_{t-1}} \\
0 & 0 & \frac{1-d-v}{1-d-v K_{t-1}}
\end{array}\right]
$$

$$
t=1,2,3, \ldots
$$

where for convenience we have written

$$
\phi_{t-1}^{(1)}=\frac{b+(a h-b g) K_{t-1}}{1-d-v K_{t-1}},
$$

$$
\phi_{t-1}^{(2)}=\frac{\gamma K_{t-1}}{1-d-v K_{t-1}} \text {. }
$$

The theorem now follows by substituting from (3.20) through (3.23) in (3.11) and simplifying.

The fundamental matrix derived in Theorem 3.2 leads directly to the following first passage characteristics: (i) first passage probabilities to states $\{(0,0)(0,-1)\}$ and $\{(\mathrm{N},+1),(\mathrm{N}, 0)\}$ while avoiding each other, (ii) mean and variance of first passage times of such transitions, and (iii) first passage probabilities into states identified in (i), but not necessarily avoiding each other. To determine the first two characteristics we convert states 0 and $N$ of (3.1) into absorbing states, and rearrange and re-partition the matrix $P$ as 


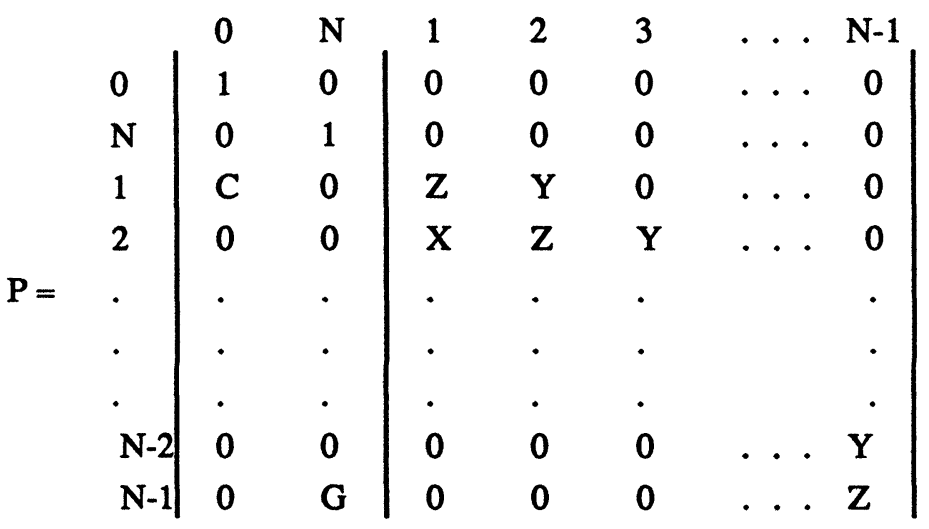

$$
\begin{aligned}
& =\left[\begin{array}{ll}
\mathrm{I} & 0 \\
\mathrm{~L} & \mathrm{H}
\end{array}\right] .
\end{aligned}
$$

The corresponding fundamental matrix is

$$
M=(\mathrm{I}-\mathrm{H})^{-1}
$$

as derived in Theorem 3.2, with $\mathrm{N}$ replaced by $\mathrm{N}-1$. Its row sums give the expected time the process takes to enter 0 or $\mathrm{N}$, having started at the corresponding state. The fundamental matrix can also be used to compute variances of first passage times (see Kemeny and Snell, 1960). The first passage probabilities to states 0 and $\mathbf{N}$ from any one of the intitial states are obtained as the elements of the matrix product ML.

Finally, to determine first passage characteristics of the process to state 0 (or $\mathrm{N}$ ), allowing the process to visit state $\mathrm{N}$ (or 0 ) in the mean time, we have to extend the matrix $\mathrm{H}$ of (3.24) as follows. 


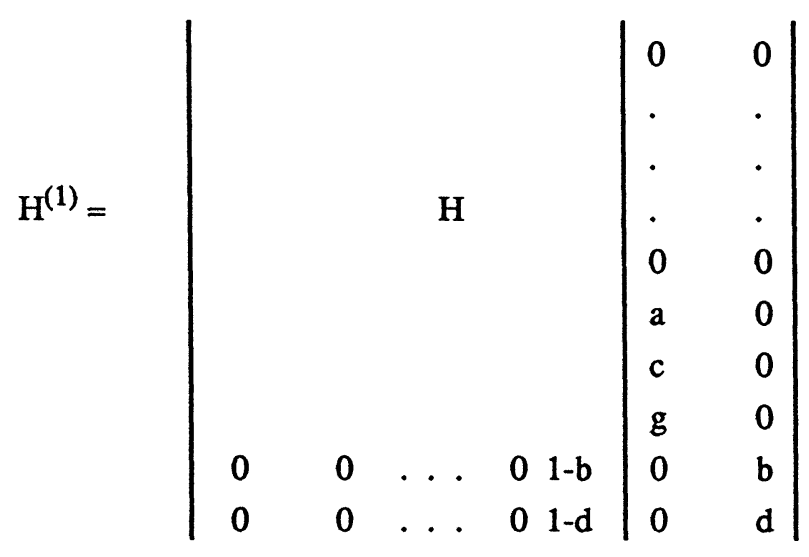

or

$$
\mathrm{H}^{(2)}=\left|\begin{array}{cc|ccccc}
\mathrm{d} & 0 & 1-\mathrm{d} & 0 & 0 & \ldots & 0 \\
\mathrm{~h} & 0 & 1-\mathrm{h} & 0 & 0 & \ldots & 0 \\
0 & 1-\mathrm{a}-\mathrm{b} & & & & & \\
0 & 1-\mathrm{c}-\mathrm{d} & & & & & \\
0 & 1-\mathrm{g}-\mathrm{h} & & & & & \\
0 & 0 & & & \mathrm{H} & & \\
. & . & & & & & \\
. & . \\
. & . \\
0 & 0 & & & & & \\
0 & 0
\end{array}\right|
$$

The fundamental matrix $M^{(1)}=\left(I-H^{(1)}\right)^{-1}$ is used while considering the first passage to state 0 , and $\mathrm{M}^{(2)}=\left(\mathrm{I}-\mathrm{H}^{(2)}\right)^{-1}$ is used while considering the first passage to state $\mathrm{N}$. In these cases the results of Theorem 3.2 can be extended using methods of Theorem 2.2 of Lal and Bhat (1988), and the elements $M_{i j}^{(1)}$ and $M_{i j}^{(2)}$ of $M^{(1)}$ and $M^{(2)}$ can be expressed in terms of elements $M_{i j}$ of equations (3.14) through (3.17). We shall not give them here due to their cumbersome nature.

The first passage results given by $M_{i j}$ of (3.14) through (3.17) may look involved and complicated. Nevertheless, as anyone well-versed in scientific computations can realize, they can be conveniently used to get useful numerical results. 


\section{REFERENCES}

[1] Bender, E.A. and Richmond, L.B. (1984), "Correlated Random Walks," Ann. Prob. 12, 274-278.

[2] Darroch, J.N. and Whitord, H.J. (1972), "Exact Fluctuation Results for Markov Dependent Coin Tossing, " J. Appl. Prob. 9, 158-168.

[3] Gillis, J. (1955), "Correlated Random Walks," Proc. Camb. Phil. Soc. 51, 639651.

[4] Gupta, H.C. (1958), "Diffusion by Discrete Movements," Sankhya 20, 295-308.

[5] Henderson, R., Renshaw, E. and Ford, D. (1983), "A Note on the Recurrence of a Correlated Random Walk", J. Appl. Prob. 20, 696-699.

[6] Henderson, R., Renshaw, E. and Ford, D. (1984), " A Correlated Random Walk Model for Two Dimensional Diffusion, " J. Appl. Prob. 21, 233-246.

[7] Jain, G. C. (1971), "Some Results in a Correlated Random Walk," Canad. Math. Bull. 14, 341-347.

[8] Jain, G.C. (1973), "On the Expected Number of Visits of a Particle Before Absorption in a Correlated Random Walk," Canad. Math. Bull. 16, 389-395.

[9] Kemeny, J.G. and Snell, J.L. (1960), Finite Markov Chains, Springer-Verlag, N.Y.

[10] Lal, R. and Bhat, U.N. (1987), "Reduced Systems in Markov Chains and Their Applications in Queueing Theory," Queueing Systems, 2, 147-172.

[11] Lal, R. and Bhat, U.N. (1988), "Reduced System Algorithms for Markov Chains," Management Sci. (to appear).

[12] Mohan, C. (1955), "The Gambler's Ruin Problem with Correlation," Biometrika 42, 486-493.

[13] Nain, R.B. and Sen, K. (1979a), "Unrestricted Correlated Random Walk with Stay," Metron, 36, 159-172.

[14] Nain, R.B. and Sen, K. (1979b), "Correlated Random Walk with Absorbing Barriers," Metron 37, 149-163.

[15] Nain, R.B. and Sen, K. (1980), "Transition Probability Matrices for Correlated Walks," J. Appl. Prob. 17, 253-258.

[16] Neuts, M.F. (1981), Matrix-Geometric Solutions in Stochastic Models, The Johns Hopkins University Press, Baltimore.

[17] Proudfoot, A.D. and Lampard, D.G. (1972), "A Random Walk Problem with Correlation," J. Appl. Prob. 9, 436-440. 
[18] Renshaw, E. and Henderson, R. (1981), "The Correlated Random Walk," J. Appl. Prob. 18, 403-414.

[19] Roerdink, J.B.T.M. (1985), "A Note on the Asymptotic Properties of Correlated Random Walks," J. Appl. Prob. 22, 951-956.

[20] Seth, A. (1963), "The Correlated Unrestricted Random Walk," J. Roy. Stat. Soc. B 25, 394-400. 


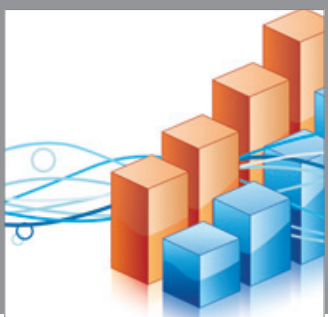

Advances in

Operations Research

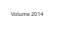

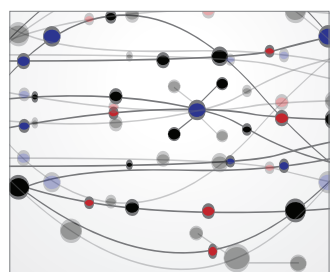

\section{The Scientific} World Journal
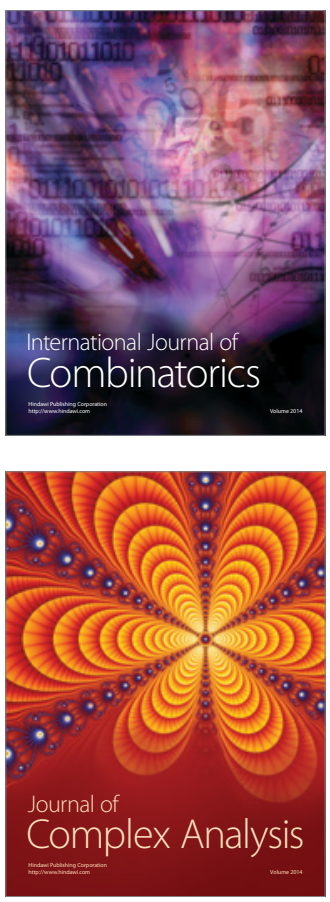

International Journal of

Mathematics and

Mathematical

Sciences
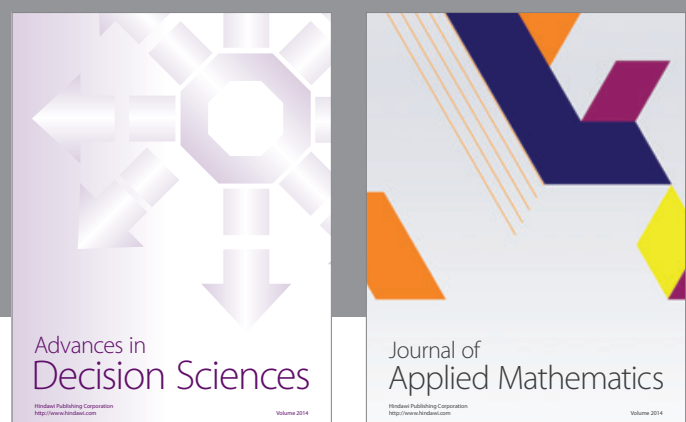

Journal of

Applied Mathematics
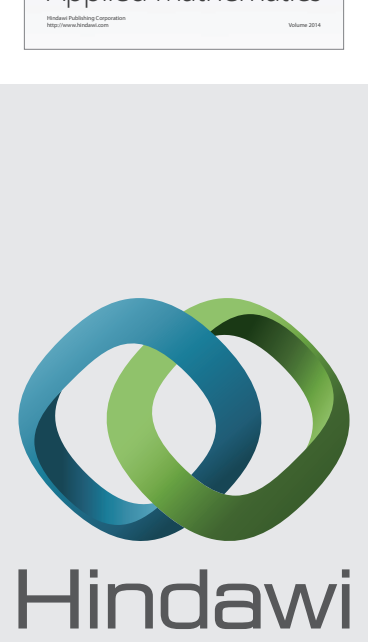

Submit your manuscripts at http://www.hindawi.com
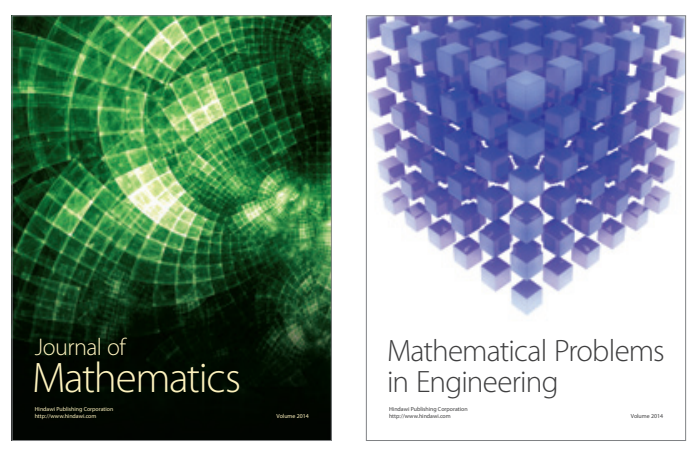

Mathematical Problems in Engineering
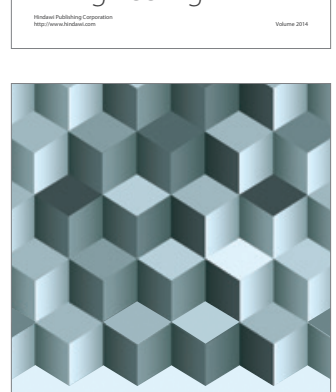

Journal of

Function Spaces
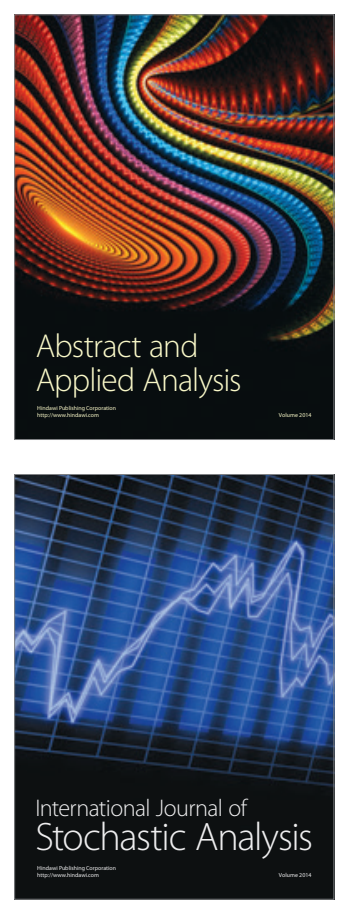

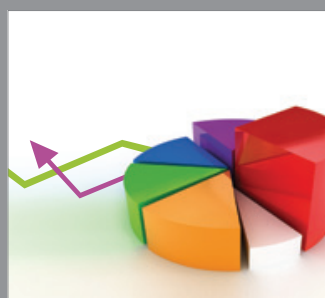

ournal of

Probability and Statistics

Promensencen
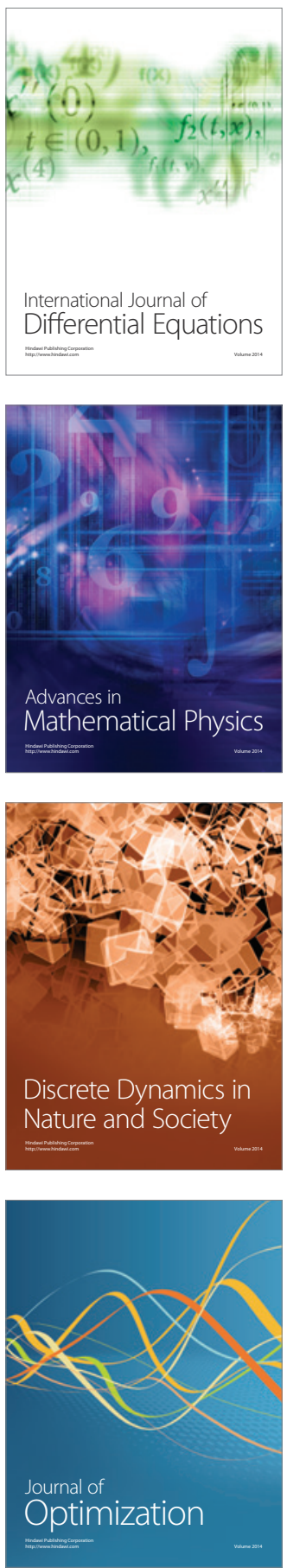